\title{
A NOTE ON A TRIGONOMETRIC MOMENT PROBLEM
}

\section{ROBERT M. YOUNG}

ABSTRACT. A sequence $\left\{\lambda_{n}\right\}_{n=-\infty}^{\infty}$ is said to be an interpolating sequence for $L^{2}(-\pi, \pi)$ if the system of equations

$$
c_{n}=\int_{-\pi}^{\pi} f(t) e^{i \lambda n^{t}} d t \quad(-\infty<n<\infty)
$$

admits a solution $f$ in $L^{2}(-\pi, \pi)$ whenever $\left\{c_{n}\right\} \in l^{2}$. If the solution is unique then $\left\{\lambda_{n}\right\}$ is said to be a complete interpolating sequence. It is shown that if the imaginary part of $\lambda_{n}$ is uniformly bounded and if $\left|\operatorname{Re}\left(\lambda_{n}\right)-n\right| \leq L<1 / 4(-\infty<n<\infty)$, then $\left\{\lambda_{n}\right\}$ is a complete interpolating sequence and $\left\{e^{i \lambda_{n} t}\right\}$ is a Schauder basis for $L^{2}(-\pi, \pi)$. It is also shown that this result is sharp in the sense that the condition $\left|\lambda_{n}-n\right|<1 / 4$ is not sufficient to guarantee that $\left\{\lambda_{n}\right\}$ is an interpolating sequence.

1. A sequence $\left\{\lambda_{n}\right\}_{n=-\infty}^{\infty}$ of (distinct) real or complex numbers is said to be an interpolating sequence for $L^{2}(-\pi, \pi)$ if the system of equations

$$
c_{n}=\int_{-\pi}^{\pi} f(t) e^{i \lambda n^{t}} d t \quad(-\infty<n<\infty)
$$

admits a solution $f$ in $L^{2}(-\pi, \pi)$ whenever $\left\{c_{n}\right\} \in l^{2}$. If, in addition, the set of exponentials $\left\{e^{i \lambda_{n} t}\right\}$ is complete in $L^{2}(-\pi, \pi)$, then the interpolation is unique and in this case we say that $\left\{\lambda_{n}\right\}$ is a complete interpolating sequence.

In this paper we are concerned with sequences which are close to the integers in the sense that $\sup \left|\lambda_{n}-n\right|<\infty$. It is shown that if the imaginary part of $\lambda_{n}$ is uniformly bounded and if $\left|\operatorname{Re}\left(\lambda_{n}\right)-n\right| \leq L<1 / 4(-\infty<n<\infty)$, then $\left\{\lambda_{n}\right\}$ is a complete interpolating sequence and $\left\{e^{i \lambda_{n} t}\right\}$ is a Schauder basis for $L^{2}(-\pi, \pi)$. It is also shown that this result is sharp in the sense that the condition

$$
\left|\lambda_{n}-n\right|<1 / 4
$$

is not sufficient to guarantee that $\left\{\lambda_{n}\right\}$ is an interpolating sequence. These results are extensions of work done by the author in [7] and [8].

Received by the editors February 18, 1974.

AMS (MOS) subject classifications (1970). Primary 42A80; Secondary 30A80.

Key words and phrases. Interpolating sequence, frames, Paley-Wiener space. 
2. The following theorem [7] gives two important properties of complete interpolating sequences which make them useful in the study of nonharmonic Fourier series.

Theorem A. Let $\left\{\lambda_{n}\right\}$ be a sequence of points lying in a strip parallel to the real axis.

(i) If $\left\{\lambda_{n}\right\}$ is a complete interpolating sequence, then the set of exponentials $\left\{e^{i \lambda} n^{t}\right\}$ is a Schauder basis for $L^{2}(-\pi, \pi)$.

(ii) If $\left\{\operatorname{Re}\left(\lambda_{n}\right)\right\}$ is a complete interpolating sequence, then $\left\{\lambda_{n}\right\}$ is a complete interpolating sequence.

It was proved by M. I. Kadec [4] that if each $\lambda_{n}$ is real and $\left|\lambda_{n}-n\right| \leq$ $L<1 / 4(-\infty<n<\infty)$, then the Paley-Wiener inequality obtains:

$$
\left\|\sum c_{n}\left(e^{i n t}-e^{i \lambda} n^{t}\right)\right\|^{2} \leq \theta^{2} \sum\left|c_{n}\right|^{2} \quad(0 \leq \theta<1) .
$$

It follows, in particular, that $\left\{\lambda_{n}\right\}$ is a complete interpolating sequence [6, pp. 100, 115]. Combining this with Theorem A yields the following result.

Theorem 1. If $\left\{\lambda_{n}\right\}$ is a sequence of points lying in a strip parallel to the real axis, and if $\left|\operatorname{Re}\left(\lambda_{n}\right)-n\right| \leq L<1 / 4(-\infty<n<\infty)$, then $\left\{\lambda_{n}\right\}$ is a complete interpolating sequence and $\left\{e^{i \lambda} n^{t}\right\}$ is a Schauder basis for $L^{2}(-\pi, \pi)$.

Remark. An incorrect proof of this result appears in [4], where a theorem of Duffin and Schaeffer is misstated.

3. Theorem 1 is sharp in the sense that $L$ cannot be taken equal to $1 / 4$. This follows from the fact that if $\left\{\mu_{n}\right\}$ is given by

$$
\begin{aligned}
\mu_{n} & =n-1 / 4, & & n>0, \\
& =n+1 / 4, & & n<0,
\end{aligned}
$$

then $\left\{\mu_{n}\right\}$ is not an interpolating sequence [3, p. 378]. We are going to prove the following stronger result.

Theorem 2. Condition (1) is not sufficient to guarantee that $\left\{\lambda_{n}\right\}$ is an interpolating sequence.

Theorem 2 extends the result, established in [8], that (1) does not imply (2). The proof of Theorem 2 will require the following lemma.

Lemma. Suppose that the set of exponentials $\left\{e^{i \lambda} n^{t}\right\}_{n=1}^{\infty}$ is complete in $L^{2}(-\pi, \pi)$. Then there exist numbers $\epsilon_{n}>0$ such that the set $\left\{e^{i \gamma_{n} t}\right\}$ is also complete in $L^{2}(-\pi, \pi)$ whenever $\left|\lambda_{n}-\gamma_{n}\right| \leq \epsilon_{n}$. 
Proof. By making a suitable translation, we may suppose that the real part of $\lambda_{n}$ is not an integral multiple of $1 / 2$. Let $K_{n}$ denote the integer closest to $\lambda_{n}$ and let $\delta_{n}=\left|\lambda_{n}-K_{n}\right|$. Choose $\epsilon_{n}\left(0<\epsilon_{n}<\delta_{n} / 2\right)$ small enough so that the following conditions are satisfied:

(i) The interval $\left[\operatorname{Re}\left(\lambda_{n}\right)-\epsilon_{n}, \operatorname{Re}\left(\lambda_{n}\right)+\epsilon_{n}\right]$ contains no number of the form $k+1 / 2$, with $k$ integral, and

(ii) $\sum_{n=1}^{\infty} \epsilon_{n} / \delta_{n}<\infty$.

We show that if $\left|\lambda_{n}-\gamma_{n}\right| \leq \epsilon_{n}(n=1,2, \ldots)$, then $\left\{e^{i \gamma_{n} t}\right\}$ is complete in $L^{2}(-\pi, \pi)$. Suppose not. Then there exists a function $h$ in $L^{2}(-\pi, \pi)$, not identically zero, such that

$$
\int_{-\pi}^{\pi} h(t) e^{i \gamma} n^{t} d t=0 \quad(n=1,2, \cdots) .
$$

Let $H$ denote the Paley-Wiener space of entire functions of exponential type $\pi$ which are square integrable on the real axis. If we set

$$
f(z)=\int_{-\pi}^{\pi} h(t) e^{i z t} d t
$$

then $f$ belongs to $H$, is not identically zero, and $f\left(\gamma_{n}\right)=0$ for each $\gamma_{n}$. We may suppose in addition that $f(0)=1$. This is clear if $f(0) \neq 0$, while if $f$ has a zero of order $m$ at the origin, then dividing $f$ by a suitable multiple of $z^{m}$ produces the desired function.

Let us set $f_{N}(z)=f(z) g_{N}(z)$, where

$$
g_{N}(z)=\prod_{n=1}^{N} \frac{\gamma_{n}}{\lambda_{n}} \cdot \frac{z-\lambda_{n}}{z-\gamma_{n}} \quad(N=1,2, \cdots) .
$$

Then $f_{N} \in H, f_{N}\left(\lambda_{k}\right)=0(k=1,2, \ldots, N)$, and $f_{N}(0)=1$. We are going to show that the norms

$$
\left\|f_{N}\right\|=\left\{\int_{-\infty}^{\infty}\left|f_{N}(x)\right|^{2} d x\right\}^{1 / 2}
$$

are uniformly bounded in $N$. Since the value of $\|f\|$, for each $f$ in $H$, is given by the formula

$$
\|f\|^{2}=\sum_{k=-\infty}^{\infty}|f(k)|^{2}
$$

it is sufficient to show that the numbers $\left|g_{N}(k)\right|$ are uniformly bounded in $N$ and $k$. By the triangle inequality, $\left|\lambda_{n}-k\right| \leq\left|\gamma_{n}-k\right|+\left|\lambda_{n}-\gamma_{n}\right|$, so that

$$
\left|\frac{k-\lambda_{n}}{k-\gamma_{n}}\right| \leq 1+\frac{\epsilon_{n}}{\left|k-\gamma_{n}\right|} \leq 1+\frac{\epsilon_{n}}{\left|K_{n}-\gamma_{n}\right|} \leq 1+\frac{\epsilon_{n}}{\delta_{n}-\epsilon_{n}} \leq 1+\frac{2 \epsilon_{n}}{\delta_{n}} .
$$


Similarly, the inequality $\left|\gamma_{n}\right| \leq\left|\gamma_{n}-\lambda_{n}\right|+\left|\lambda_{n}\right|$ shows that

$$
\left|\gamma_{n} / \lambda_{n}\right| \leq 1+\epsilon_{n} /\left|\lambda_{n}\right| \leq 1+2 \epsilon_{n} / \delta_{n}
$$

Therefore, for all $N$ and $k$,

$$
\left|g_{N}(k)\right| \leq \prod_{n=1}^{N}\left(1+\frac{2 \epsilon_{n}}{\delta_{n}}\right)^{2} \leq \prod_{n=1}^{\infty}\left(1+\frac{2 \epsilon_{n}}{\delta_{n}}\right)^{2} \leq \exp \left(\sum_{n=1}^{\infty} \frac{4 \epsilon_{n}}{\delta_{n}}\right),
$$

and hence, $\sup _{N}\left\|f_{N}\right\|<\infty$.

Since $H$ is a functional Hilbert space, it follows that a subsequence of $\left\{f_{N}\right\}$ will converge weakly to a function $F$ in $H$ for which $F\left(\lambda_{n}\right)=0(n=1$, $2, \ldots)$, and $F(0)=1$. It follows from the representation theorem of Paley and Wiener for functions in $H[1$, p. 103] that

$$
F(z)=\int_{-\pi}^{\pi} g(t) e^{i z t} d t
$$

for some function $g$ in $L^{2}(-\pi, \pi)$. Therefore, the set $\left\{e^{i \lambda} n^{t}\right\}$ is not complete in $L^{2}(-\pi, \pi)$, contrary to assumption. The contradiction establishes the lemma.

We can now establish Theorem 2.

Proof of Theorem 2. Let us suppose to the contrary that condition (1) is sufficient to ensure that $\left\{\lambda_{n}\right\}$ is an interpolating sequence. If $\left\{\mu_{n}\right\}$ is given by (3), then the set of exponentials $\left\{e^{i \mu_{n} t}\right\}$ is complete in $L^{2}(-\pi, \pi)[5$, p. 67]. It follows from the lemma that a sequence $\left\{\lambda_{n}\right\}$ can be chosen so that $\lambda_{n}-\mu_{n} \rightarrow 0(n \rightarrow \pm \infty),\left\{e^{i \lambda} n^{t}\right\}$ is complete in $L^{2}(-\pi, \pi)$, and (1) holds. Let us define a mapping $T: L^{2}(-\pi, \pi) \rightarrow l^{2}$ by

$$
T(f)=\left\{\int_{-\pi}^{\pi} f(t) e^{i \lambda n^{t}} d t\right\} .
$$

It follows from the fact that $\left\{\lambda_{n}\right\}$ is separated, that is, $\left|\lambda_{m}-\lambda_{n}\right| \geq \delta>0(m$ $\neq n)$, and the imaginary part of $\lambda_{n}$ is uniformly bounded, that $T$ is a continuous linear mapping into $l^{2}$. Since $\left\{\lambda_{n}\right\}$ is an interpolating sequence, $T$ is in fact onto $l^{2}$. The completeness of the exponentials $\left\{e^{i \lambda n^{t}}\right\}$ shows that $T$ is also 1-1. It follows from the open mapping theorem that $T$ has a continuous inverse. Thus, there exist positive constants $A$ and $B$ such that

$$
A\|f\|^{2} \leq \sum_{n}\left|\int_{-\pi}^{\pi} f(t) e^{i \lambda n^{t}} d t\right|^{2} \leq B\|f\|^{2}
$$

for every function $f$ in $L^{2}(-\pi, \pi)$. In the terminology of Duffin and Schaeffer [2], the set $\left\{e^{i \lambda n^{t}}\right\}$ is a frame. We complete the proof by showing that this leads to a contradiction. 
It was shown in [8] that if $\left\{e^{i \lambda_{n} t}\right\}$ is a frame and if $\left\{\beta_{n}\right\}$ is any sequence of complex numbers for which $\lambda_{n}-\beta_{n} \rightarrow 0(n \rightarrow \pm \infty)$, then $\left\{e^{i \beta_{n} t}\right\}$ is either a frame or an incomplete set. But $\left\{e^{i \mu_{n} t}\right\}$ is not a frame [8], and since it also fails to be incomplete, we can only conclude that $\lambda_{n}-\mu_{n}$ must not approach zero. However, this contradicts the choice of $\left\{\lambda_{n}\right\}$, and the theorem is established.

Remark. It is well known that the condition $\lambda_{n+1}-\lambda_{n} \geq \gamma>1(-\infty<n$ $<\infty)$ implies that $\left\{\lambda_{n}\right\}$ is an interpolating sequence $[3$, p. 368]. The proof of Theorem 2, with obvious modifications, shows however that the weakened condition $\lambda_{n+1}-\lambda_{n}>1$ is not sufficient for $\left\{\lambda_{n}\right\}$ to be an interpolating sequence.

\section{REFERENCES}

1. R. P. Boas, Jr., Entire functions, Academic Press, New York, 1954. MR $16,1914$.

2. R. J. Duffin and A. C. Schaeffer, A class of nonharmonic Fourier series, Trans. Amer. Math. Soc. 72 (1952), 341-366. MR 13, 839.

3. A. E. Ingham, Some trigonometrical inequalities with applications to the theory of series, Math. Z. 41 (1936), 367-379.

4. M. I. Kadec, The exact value of the Paley-Wiener constant, Dokl. Akad. Nauk SSSR 155 (1964), 1253-1254 = Soviet Math. Dokl. 5 (1964), 559-561. MR 28 \#5289.

5. N. Levinson, Gap and density theorems, Amer. Math. Soc. Colloq. Publ., vol. 26, Amer. Math. Soc., Providence, R. I., 1940. MR 2, 180.

6. N. Wiener and R. E. A. C. Paley, Fourier transforms in the complex domain, Amer. Math. Soc. Colloq. Publ., vol, 19, Amer. Math. Soc., Providence, R. I., 1934.

7. R. M. Young, Interpolation in a classical Hilbert space of entire functions, Trans. Amer. Math. Soc. 192 (1974), 97-114.

8. - Inequalities for a perturbation theorem of Paley and Wiener, Proc. Amer. Math. Soc. 43 (1974), 320-322. 